\title{
Knockdown of lncRNA SNHG4 suppresses gastric cancer cell proliferation and metastasis by targeting miR-204-5p
}

\author{
Xian-Bin CHENG ${ }^{1}$, Tao ZHANG ${ }^{2}$, Hong-Jing ZHU³ , Ning MA, Xiao-Dan SUN ${ }^{5}$, Shou-Han WANG ${ }^{6}$, Yang JIANG ${ }^{1, *}$ \\ ${ }^{1}$ Department of Gastrointestinal Colorectal and Anal Surgery, China-Japan Union Hospital of Jilin University, Changchun, Jilin, China; ${ }^{2}$ School \\ of Pharmaceutical Sciences, Changchun University of Chinese Medicine, Changchun, Jilin, China; ${ }^{3}$ Department of Pediatric Ultrasound, The \\ First Hospital of Jilin University, Changchun, Jilin, China; ${ }^{4}$ Department of Hepatopancreatobiliary Surgery, China-Japan Union Hospital of Jilin \\ University, Changchun, Jilin, China; ${ }^{5}$ Department of Second Gynecologic Oncology Surgery, Jilin Cancer Hospital, Changchun, Jilin, China; \\ ${ }^{6}$ Department of Abdominal Oncology Surgery, Jilin Cancer Hospital, Changchun, Jilin, China
}

*Correspondence: jiangyang@jlu.edu.cn

Received September 14, 2020 / Accepted January 14, 2021

\begin{abstract}
Long non-coding RNAs (lncRNAs) have been identified as critical regulators in gastric cancer (GC) progression. However, whether lncRNA small nucleolar host gene 4 (SNHG4) functions in GC development remains unknown. In this study, the bio-functional role of SNHG4 and its potential mechanism on GC progression were systematically dissected. To investigate the role of SNHG4 in GC, we silenced SNHG4 using short hairpin RNAs (shRNAs) to perform loss-of-function assays. The results showed that SNHG4 expression in GC cells was at a higher level compared to normal gastric mucosal epithelial cells. Knockdown of SNHG4 dramatically suppressed proliferation, migration and invasion, and blocked cell cycle progression of GC cells. Moreover, knockdown of SNHG4 upregulated microRNA-204-5p (miR-204-5p) expression, whereas downregulated ribonucleotide reductase subunit M2 (RRM2) expression in GC cells. Dual-luciferase reporter assay results showed that miR-204-5p was a direct target of SNHG4. Additionally, knockdown of SNHG4 suppressed GC tumorigenesis in xenograft mouse models. Taken together, these data demonstrated that knockdown of SNHG4 suppressed GC development by targeting miR-204-5p.
\end{abstract}

Key words: SNHG4, miR-204-5p, gastric cancer, proliferation, metastasis

Gastric cancer (GC) is one of the most aggressive and prevalent malignancies worldwide with an estimation of $1,033,701$ new cases and 782,685 cancer-related deaths in 2018 [1]. Among all populations, the burden of GC is very high in countries from Asia, Latin America, and Central and Eastern Europe [2]. Despite some advances that have been made in revealing the epidemiology, pathology, and therapeutic options of GC, it remains one of the biggest threats of cancer mortality [3]. The reason for the high mortality of GC may be that the diagnosis of GC patients frequently occurs at an advanced stage, which severely impedes the effectiveness of therapy [4]. Hence, investigations on the available screening approaches for early GC remain critical for preventing and treating GC.

It has been reported that the accumulation of genetic and epigenetic alternations is a major incentive for cancer pathogenesis [5]. Non-coding RNAs (ncRNAs), such as long non-coding RNAs (lncRNAs) and microRNAs (miRNAs), have been confirmed to affect the progression, growth, and spread of cancers [6-8]. Among them, small nucleolar host gene 4 (SNHG4) is a novel lncRNA that belongs to the SNHGs family, members of which have been documented to play critical roles in regulating tumorigenesis [9-11]. Recent studies have identified that SNHG4 functions as an important carcinogenic molecule in several cancers. For instance, $\mathrm{Xu}$ et al. reported that SNHG4 facilitates tumor growth by sponging miR-224-3p and is regarded as a poor prognostic factor in human osteosarcoma [12]. Li et al. demonstrated that SNHG4 acts as a competing endogenous RNA (ceRNA) for sponging miR-148a-3p, thereby upregulating c-Met expression, to promote cervical cancer progression [13]. However, the role of SNHG4 in GC development has not been elucidated.

Emerging evidence has demonstrated that lncRNAs and miRNAs interact with each other to regulate tumorigenesis and metastasis [14]. By using an online database for preliminary examination, we found that there is the binding potential between SNHG4 and miR-204-5p. miR-204-5p has been reported to function in retarding GC progression previously $[15,16]$. Recently, Wu et al. demonstrated that 
SNHG4 promotes tumorigenesis and invasion by acting as a ceRNA to sponge miR-204-5p and upregulate runt-related transcription factor 2 (RUNX2) expression in renal cell carcinoma [17]. Nevertheless, whether SNHG4 affects the GC progression by regulating miR-204-5p is still unknown. In this study, we investigated the effect of SNHG4 on the GC progression. Mechanically, we demonstrated that SNHG4 affects the GC progression by regulating miR-204-5p expression.

\section{Materials and methods}

Ethics statement. The animal experiments in this work were approved by the Experimental Animal Ethics Committee of the Changchun University of Chinese Medicine (approval No. 2020217). All procedures were carefully performed in accordance with the National Institutes of Health Guide for the Care and Use of Laboratory Animals (NIH Publications No. 8023, revised 1978).

Cell lines and cell culture. Human gastric mucosal epithelial cell line GES-1 (Cat. No. ZQ0905), human gastric cancer cell lines AGS (Cat. No. ZQ0240), HGC-27 (Cat. No. ZQ0192), NCI-N87 (Cat. No. ZQ0060), and MKN-45 (Cat. No. ZQ0457), and human embryonic kidney epithelial cell line 293T (Cat. No. ZQ0033) were all obtained from Shanghai Zhongqiao Xinzhou Biotechnology Co., Ltd (China). GES-1 cells were cultivated in Dulbecco's modification of Eagle's medium (DMEM; Gibco Inc., USA) supplemented with 10\% fetal bovine serum (FBS; Zhejiang Tianhang Biotechnology Co., Ltd., China) and 1\% penicillin-streptomycin solution. AGS cells were cultivated in F12K medium (Zhongqiao Xinzhou) supplemented with 10\% FBS. NCI-N87 cells were cultivated in RPMI-1640 medium (Gibco) supplemented with $10 \%$ FBS. HGC-27 and MKN-45 cells were cultivated in RPMI-1640 medium supplemented with 20\% FBS. 293T cells were cultivated in DMEM supplemented with 10\% FBS. All cells were cultured at $37^{\circ} \mathrm{C}$ in a humidity-controlled atmosphere with $95 \%$ air and $5 \% \mathrm{CO}_{2}$.

Quantitative real-time PCR (qRT-PCR). Total RNAs in cells and xenograft tumor tissues were isolated using a highpurity total RNA rapid extraction kit (Wuxi BioTeke Co., Ltd., China). RNAs were reversely transcribed into cDNAs using M-MLV reverse transcriptase (Takara Bio Inc., Japan). qRT-PCR was conducted with Taq HS Perfect Mix (Takara) and SYBR Green (BioTeke) in a fluorescence quantitative PCR instrument (Exicycler 96; Bioneer Corporation, South Korea). The reaction conditions were as follows: initial at $94^{\circ} \mathrm{C}$ for $5 \mathrm{~min}$, denaturation at $94^{\circ} \mathrm{C}$ for $30 \mathrm{~s}$, annealing at $60^{\circ} \mathrm{C}$ for $30 \mathrm{~s}$, and extension at $72^{\circ} \mathrm{C}$ for $40 \mathrm{~s}$. The results were analyzed using the $2^{-\triangle \Delta C T}$ method. $\beta$-actin was used as an endogenous control for SNHG4 and mRNAs. U6 was used as an endogenous control for miR-204-5p. The primers were as follows: SNHG4 (forward: 5'-GGCTAGAGTACAGTGGCTCG-3'; reverse: 5'-GCAAATCGCAAGGTCAGG-3'); ribonucleotide reductase subunit M2 (RRM2; forward: 5'-GGGAT-
GAATTGCACTCTAA-3'; reverse: 5'-TCCTCTGATACTCGCCTACT-3'); $\beta$-actin (forward: 5'-GGCACCCAGCACAATGAA-3'; reverse: 5'-TAGAAGCATTTGCGGTGG-3'); miR-204-5p (forward: 5'-TTCCCTTTGTCATCCTATGCCT-3'; reverse: 5'-GCAGGGTCCGAGGTATTC-3'); U6 (forward: 5'-GCTTCGGCAGCACATATACT-3'; reverse: 5'-GCAGGGTCCGAGGTATTC-3'). This test was independently performed in triplicate.

Cell transfection. For knockdown of SNHG4, short hairpin RNAs (shRNAs) targeting human SNHG4 (NR_003141.3) and scramble control (NC) were designed, synthesized, and cloned into pRNAH1.1 vector (Nanjing Jinsirui Biotechnology Co., Ltd., China). Sequences for shRNAs were as follows: shNC (5'-GATCCCCTTCTCCGAACGTGTCACGTTTCAAGAGAACGTGACACGTTCGGAGAATTTTT-3'); shSNHG4-1 (5'-GGCTCCACTAGGACACACAGATTTCAAGAGAATCTGTGTGTCCTAGTGGAGCTTTTT-3'); shSNHG4-2 (5'-GGAATGACATCTACCTCCATCATTCAAGAGATGATGGAGGTAGATGTCATTCTTTTT-3'). HGC-27 and MKN-45 cells at a confluence of $80 \%$ were transfected using Lipofectamine $\mathrm{T}^{\mathrm{TM}} 3000$ transfection reagent (Invitrogen Corporation, USA) according to the manufacturer's instructions. G418 (100 $\mu \mathrm{g} / \mathrm{ml}$, NanJing KeyGene Biotech Co., Ltd., China) was used to screen stable transfection clones. Transfection efficacy was evaluated using qRT-PCR.

For overexpression or knockdown of miR-204-5p, hsa-miR-204-5p mimics, or hsa-miR-204-5p inhibitor and their NCs (Shanghai GenePharma Co., Ltd., China) were transiently transfected into cells using Lipofectamine ${ }^{\mathrm{TM}} 3000$ transfection reagent according to the manufacturer's instructions. hsa-miR-204-5p mimics, hsa-miR-204-5p inhibitor, and their NC sequences were as follows: hsa-miR-204-5p mimics (5'-UUCCCUUUGUCAUCCUAUGCCU-3' and 5'-GCAUAGGAUGACAAAGGGAAUU-3'); mimics NC (5'-UUCUCCGAACGUGUCACGUTT-3' and 5'-ACGUGACACGUUCGGAGAATT-3'); hsa-miR-204-5p inhibitor (5'-AGGCAUAGGAUGACAAAGGGAA-3'); inhibitor NC (5'-CAGUACUUUUGUGUAGUACAA-3').

Cell counting kit-8 assay. Cell viability of the transfected cells was evaluated using cell counting kit-8 (CCK-8; Sigma-Aldrich Corporation, USA) assay according to the manufacturer's instructions. Optical density (OD) values at $570 \mathrm{~nm}$ were detected using a microplate reader (ELX-800; BioTek Instruments, Inc., USA). This test was independently performed in triplicate.

Flow cytometry. Flow cytometry was performed to monitor the distribution of the cell cycle. Briefly, stably transfected cells were seeded, washed, fixed, and then incubated with a cell cycle detection kit (Shanghai Beyotime Biotechnology Co., Ltd., China). Cell cycle distribution was examined using a flow cytometer (NovoCyte; ACEA Biosciences Inc., USA) and analyzed using NovoExpress. This test was independently performed in triplicate.

Western blot. Total proteins in cells and xenograft tumor tissues were isolated using powerful radio-immunoprecipi- 
tation assay (RIPA) lysis solution (Beyotime) with phenylmethanesulfonyl fluoride (PMSF; Beyotime). The concentrations of proteins were determined by a bicinchoninic acid (BCA) assay kit (Beyotime). The same number of proteins were electrophoresed and then transferred to polyvinylidene fluoride (PVDF) membranes (Thermo Fisher Scientific Inc., USA). After blocking with $5 \%$ bovine serum albumin (BSA; Biosharp, China), the membranes were probed with cyclin D1 antibody (Cat. No. A19038, Abclonal Technology, China), cyclin-dependent kinase 6 (CDK6) antibody (Cat. No. A1545, Abclonal), matrix metallopeptidase 2 (MMP2) antibody (Cat. No. 10373-2-AP, Proteintech Group Inc., China), matrix metallopeptidase 9 (MMP9) antibody (Cat. No. 10375-2-AP, Proteintech), RRM2 antibody (Cat. No. A3424, Abclonal), and $\beta$-actin antibody (Cat. No. 600081-Ig, Proteintech). After washing with Tris buffer solution containing Tween-20 (TBST) three times, the membranes were coupled with goat anti-rabbit IgG (Cat. No. SA00001-2, Proteintech) or goat anti-mouse IgG (Cat. No. SA00001-1, Proteintech). Enhanced chemiluminescence (ECL) solution (7Sea Biotech, China) was used to visualize the blots. Densitometric analysis was conducted by the ImageJ software. This test was independently performed in triplicate.

Wound healing assay. Cell migration was evaluated using a wound healing assay. Briefly, cells at a proper confluence were treated with $1 \mu \mathrm{g} / \mathrm{ml}$ mitomycin-C (Sigma) in a serum-free medium for $1 \mathrm{~h}$. For the investigation of miR-204-5p function, NC inhibitor or miR-204-5p inhibitor was transiently transfected into the stably transfected cells and incubated for $24 \mathrm{~h}$ before the cells were treated with mitomycin-C. Subsequently, a scratch was made with a $200 \mu \mathrm{l}$ pipette tip. The images were photographed at the same position at $0 \mathrm{~h}$ and $24 \mathrm{~h}$ after the scratch was established under an inverted phase-contrast microscope (IX53; Olympus Corporation, Japan). This test was independently performed in triplicate.

Transwell assay. Cell invasion was evaluated using Transwell assay. Briefly, stably transfected cells were seeded in the upper chamber of the Matrigel-coated Transwell chamber (Corning Inc. USA), while a medium containing $30 \%$ FBS was added to the lower chamber. Twenty-four hours after cells were incubated, the chamber was fixed, stained with crystal violet (Amresco Inc., USA), and then counted under an inverted phase-contrast microscope. This test was independently performed in triplicate.

Dual-luciferase reporter gene assay. The wild-type (wt) or mutant-type (mut) sequences of human SNHG4 were cloned into pmirGLO (Jinsirui) luciferase reporter vectors. The luciferase reporter vectors and miR-204-5p mimic or NC mimic were co-transfected using Lipofectamine ${ }^{\mathrm{TM}} 3000$ transfection reagent according to the manufacturer's instructions. Forty-eight hours after transfection, luciferase activities were measured using a dual-luciferase assay kit (KeyGene). Relative luciferase (Firefly luciferase/Renilla luciferase) activity was calculated.
Xenograft tumor model. Six-week-old female BALB/c nude mice were purchased from Beijing HFK Bioscience Co. Ltd. (China) and fed with standard food and water in the specific pathogen-free house under a humidity-controlled atmosphere with $12 \mathrm{~h}$ light/12 h dark cycle at a controlled temperature. After bred adaptively for a week, mice were randomly and equally allocated to four groups ( $\mathrm{n}=6$ mice/ group). A total of $5 \times 10^{6}$ stably transfected (shNC and shSNHG4-2) HGC-27 and MKN-45 cells were subcutaneously injected into the right armpit of each nude mouse according to the group information. Twenty-five days after injected, the mice were euthanized by cervical dislocation, and the xenograft tumor tissues were carefully removed. The tumor tissues were immediately photographed under a digital camera. After the tumor tissues were weighed, a part of each tumor tissue was fixed with $4 \%$ paraformaldehyde for histopathological examinations and the rest was stored at $-80^{\circ} \mathrm{C}$ for examination.

Immunohistochemistry. The fixed tumor tissues were embedded in paraffin and sectioned into $5 \mu \mathrm{m}$ thick pieces. Then the paraffin-embedded tissue sections were dewaxed, rinsed, and rehydrated. After the antigen retrieval was performed, the sections were immunostained with anti-Ki67 (Abclonal) at $4{ }^{\circ} \mathrm{C}$ overnight. After washing with phosphatebuffered solution (PBS) twice, the sections were covered with goat anti-rabbit IgG (Thermo Fisher) for $30 \mathrm{~min}$. Diaminobenzidine (DAB) chromogen solution (Beijing Solarbio Science \& Technology Co., Ltd., China) was added for color development. The staining results were observed under a light microscope (BX53; Olympus).

Statistical analysis. Data were analyzed using GraphPad Prism software. Significant differences between the two groups were calculated by Student's t-test. Significant differences among the groups (three or more) were calculated by one-way analysis of variance (one-way analysis of variance). A p-value $<0.05$ was considered statistically significant.

\section{Results}

lncRNA SNHG4 was upregulated in human GC cells. First, the expressions of lncRNA SNHG4 in human GC cell lines (AGS, HGC-27, NCI-N87, and MKN-45) and a human normal gastric mucosal epithelial cell line (GES-1) were assessed. Its expressions in HGC-27, NCI-N87, and MKN-45 cells were significantly higher than that in GES-1 cells, while its expression in AGS cells was higher only on average, but with no significance (Figure 1A). Based on this expression pattern, we chose the two highest SNHG4 expression cell lines HGC-27 and MKN-45 to verify the role of SNHG4 on GC progression. Subsequently, stable SNHG4 knockdown HGC-27 and MKN-45 cells were constructed using shRNA (Figures 1B, 1C) to investigate the biological function of SNHG4.

Knockdown of IncRNA SNHG4 inhibits GC cell proliferation in vitro. To explore the effect of SNHG4 in GC 
A

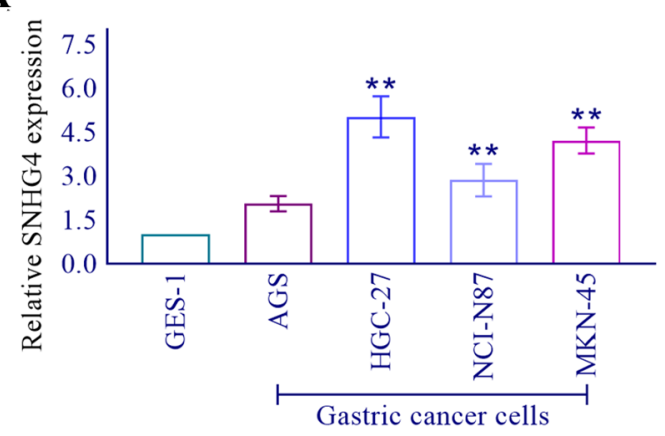

B

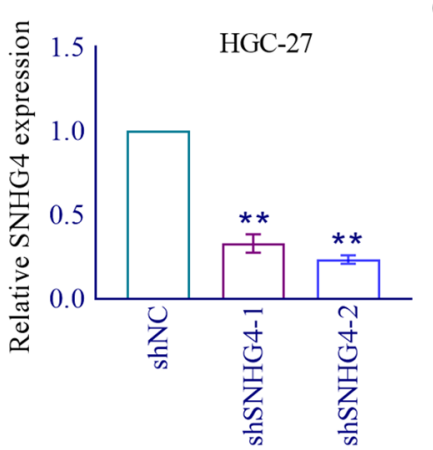

C

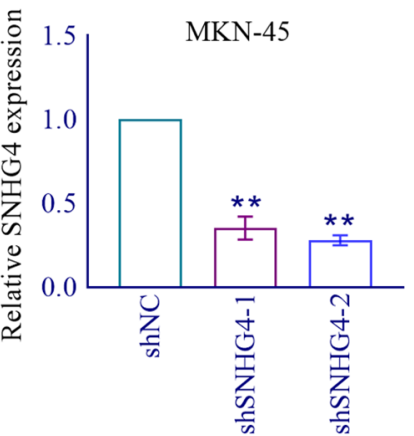

Figure 1. Expressions of SNHG4 in human GC cell lines and transfection efficiency of shRNAs targeting SNHG4. A) The relative SNHG4 expressions in human gastric mucosal epithelial cell line GES-1 and human GC cell lines AGS, HGC-27, NCI-N87, and MKN-45 were detected using qRT-PCT. B, C) The transfection efficiencies of two shRNAs targeting SNHG4 in HGC-27 cells (B) and MKN-45 cells (C) were detected using qRT-PCR. ${ }^{\star *}$ p $<0.01$

A

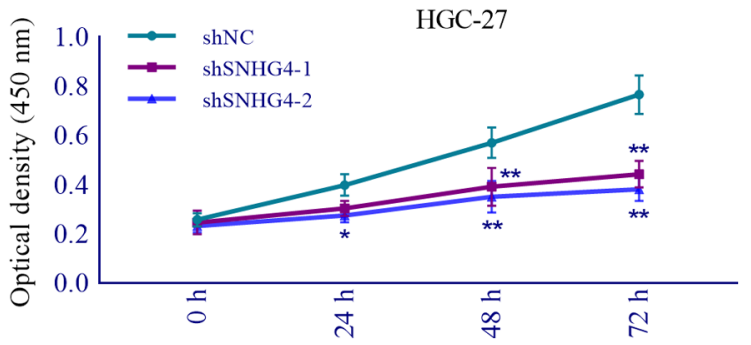

C

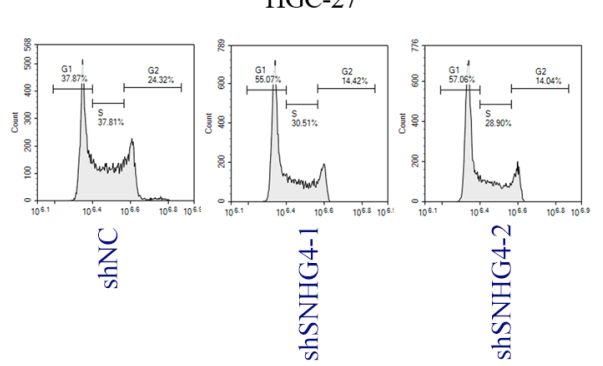

F

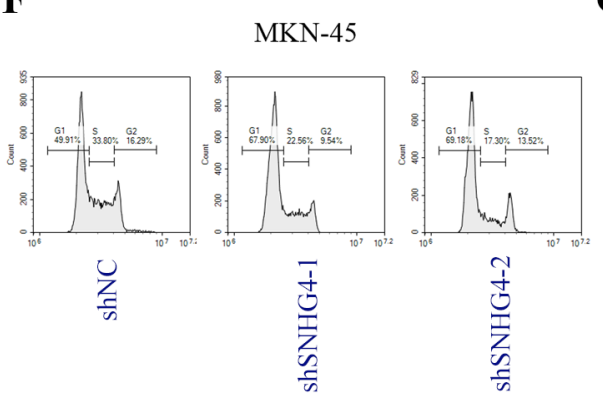

D
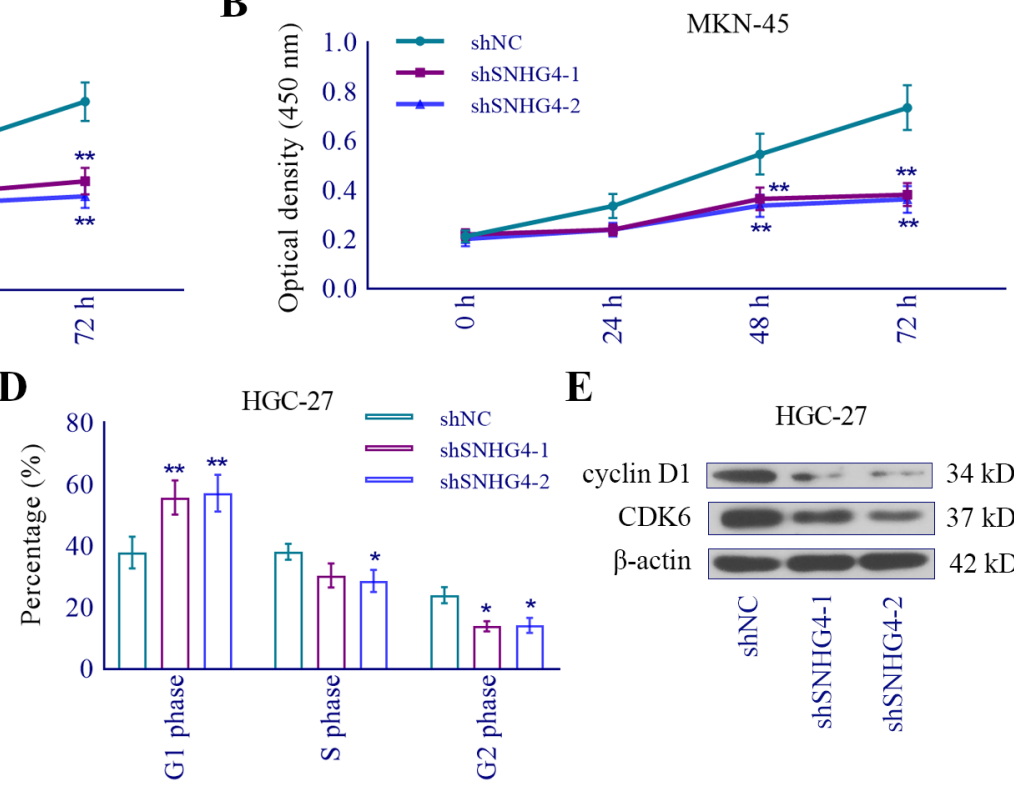

$\mathbf{E}$
CDK6

$\beta$-actin

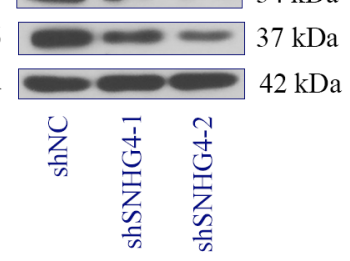

HGC-27
G

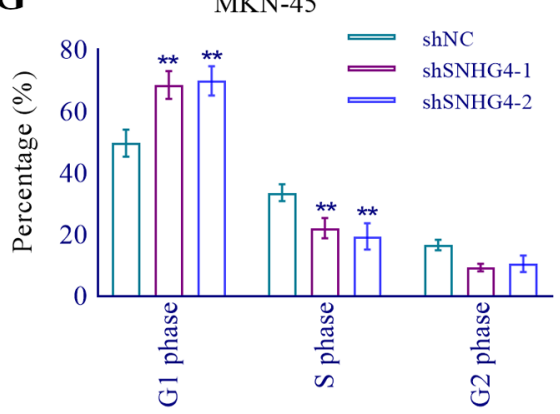

H

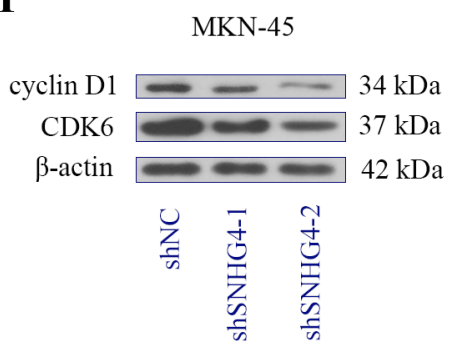

Figure 2. Knockdown of SNHG4 inhibits GC cell proliferation in vitro. A, B) Knockdown of SNHG4 inhibits cell proliferation in HGC-27 cells (A) and MKN-45 cells (B), as assessed by the CCK-8 assay. C, D) Knockdown of SNHG4 increases the cell numbers in the G1 phase and decreases the cell numbers in the S phase and G2 phase in HGC-27 cells, as assessed by flow cytometry. E) Knockdown of SNHG4 decreases cyclin D1 and CDK6 expressions in HGC-27 cells, as assessed by western blot. F, G) Knockdown of SNHG4 increases the cell numbers in the G1 phase and decreases the cell numbers in the $\mathrm{S}$ phase and G2 phase in MKN-45 cells, as assessed by flow cytometry. H) Knockdown of SNHG4 decreases cyclin D1 and CDK6 expressions in MKN-45 cells, as assessed by western blot. ${ }^{*} \mathrm{p}<0.05 ;{ }^{* *} \mathrm{p}<0.01$ 
cell proliferation, CCK-8 assay and flow cytometry were applied to assess GC cell viability and cell cycle distribution, respectively. The results of the CCK- 8 assay showed that the optical density values at $450 \mathrm{~nm}$ in shSNHG4-transfected GC cells were significantly lower than that in shNC-transfected GC cells (Figures 2A, 2B). The results of flow cytometry presented that the number of cells in the G1 phase was significantly higher, whereas in S phase and G2 phase were much lower in shSNHG4-transfected GC cells than that in the shNC-transfected GC cells (Figures 2C, 2D, 2F, and 2G). Additionally, the expressions of cyclin D1 and CDK6 were also found downregulated in shSNHG4-transfected GC cells compared with shNC-transfected GC cells (Figures 2E, 2H).

Knockdown of IncRNA SNHG4 inhibits GC cell metastasis in vitro. To explore the effect of SNHG4 in GC cell metastasis, a wound-healing assay and Transwell assay were used to assess GC cell migration ability and invasion ability, respectively. As shown in Figure 3, knockdown of SNHG4 remarkably inhibited the migration rate and invasion rate of GC cells compared to its vector control. Furthermore, knockdown of SNHG4 affected the expressions of metastasisrelated proteins, including MMP2 and MMP9, in GC cells.

miR-204-5p is a target miRNA of SNHG4. By using the bioinformatics prediction software (http://starbase.sysu.edu. cn/index.php), we predicted that miR-204-5p potentially bound to SNHG4. Hence, we detected miR-204-5p expression in several GC cell lines and also in shSNHG4-transfected GC cells. The results showed that miR-204-5p expression was significantly lower in GC cell lines (AGS, HGC-27, NCI-N87, and MKN-45) compared to a normal gastric mucosal epithelial cell line (GES-1) (Figure 4A). Also, miR-204-5p expression was significantly upregulated in shSNHG4-transfected GC cells compared to shNC-transfected GC cells (Figures 4B, $4 C)$. In addition, the results of dual-luciferase reporter gene
A
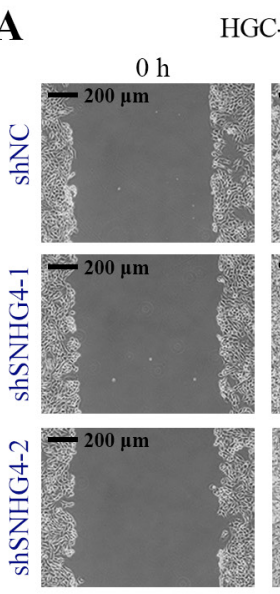

$\mathbf{E}$

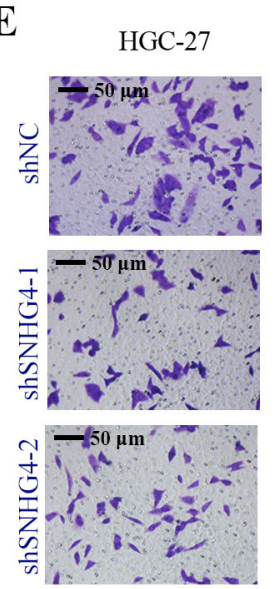

B

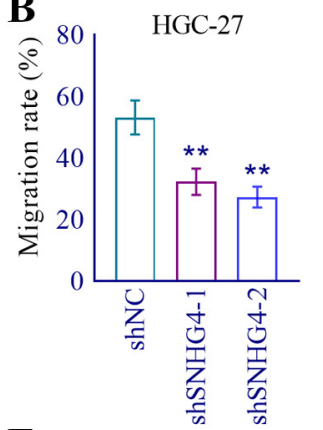

F

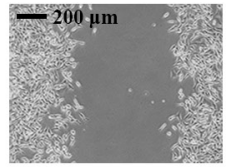

G
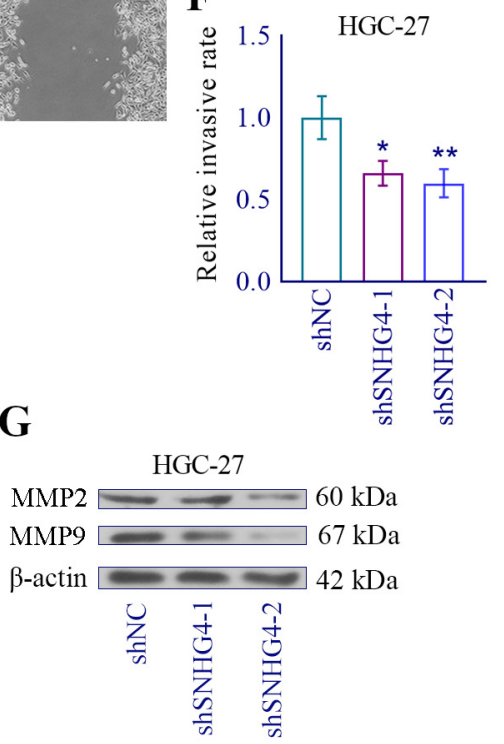

C

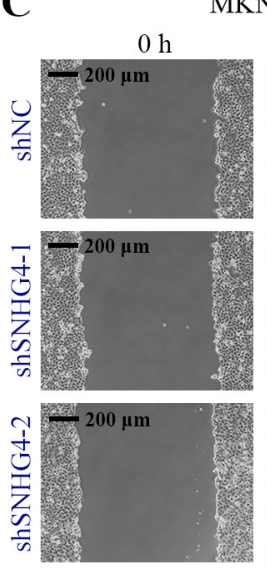

H
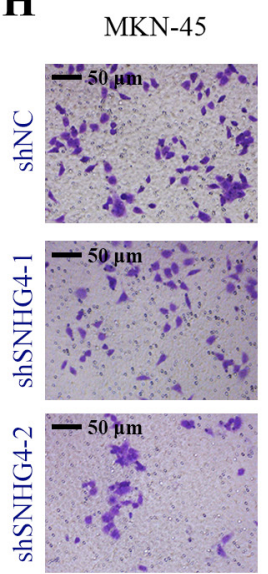
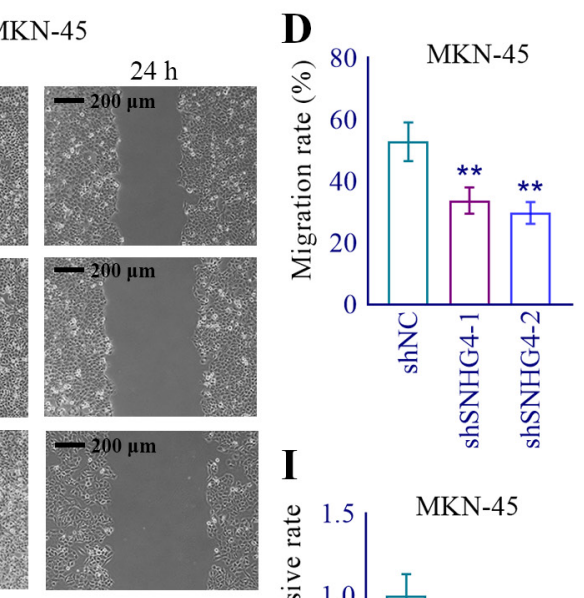

I

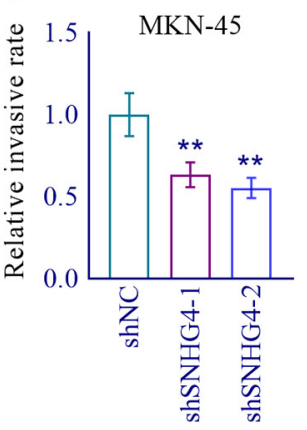

$\mathbf{J}$

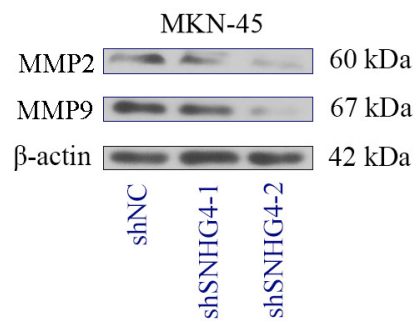

Figure 3. Knockdown of SNHG4 inhibits GC cell metastasis in vitro. A, B) Knockdown of SNHG4 inhibits cell migration in HGC-27 cells, as assessed by the wound-healing assay. C, D) Knockdown of SNHG4 inhibits cell migration in MKN-45 cells, as assessed by the wound-healing assay. E, F) Knockdown of SNHG4 inhibits cell invasion in HGC-27 cells, as assessed by the Transwell assay. G) Knockdown of SNHG4 decreases MMP2 and MMP9 expressions in HGC-27 cells, as assessed by western blot. H, I) Knockdown of SNHG4 inhibits cell invasion in MKN-45 cells, as assessed by the Transwell assay. J) Knockdown of SNHG4 decreases MMP2 and MMP9 expressions in MKN-45 cells, as assessed by western blot. ${ }^{\star}$ p $<0.05$; ${ }^{\star *}$ p $<0.01$ 
assay validated that miR-204-5p mimic dramatically reduced the luciferase activity of wt-SNHG4, while had no influence on the luciferase activity of mut-SNHG4 (Figures 4D, 4E). Furthermore, we detected the expression of RRM2, which is a known target gene of miR-204-5p. We found that RRM2 was downregulated in shSNHG4-transfected GC cells compared to shNC-transfected GC cells (Figures $4 \mathrm{~F}-4 \mathrm{I}$ ). These results indicated that SNHG4 may sponge miR-204-5p and upregulate RRM2 expression in GC cells.

miR-204-5p inhibition promotes cell proliferation and metastasis in SNHG4-knockdown GC cells. To further investigate whether SNHG4 affects GC development by regulating miR-204-5p, the miR-204-5p inhibitor was added in shSNHG4-transfected GC cells to block the inhibitory effect of miR-204-5p on GC progression. As shown in Figure 5, compared to the NC inhibitor treatment, miR-204-5p inhibitor treatment dramatically promoted cell proliferation, migration, and invasion abilities in shSNHG4transfected HGC-27 and MKN-45 cells.

Knockdown of lncRNA SNHG4 inhibits the GC cell growth in vivo. Furthermore, we investigated the bio-functional role of SNHG4 in tumorigenesis in vivo. The results showed that in the xenograft tumor mice model, the growth of shSNHG4-transfected GC cells was obviously slower compared to shNC-transfected GC cells, as reflected by a significant decrease in tumor volume and tumor weight (Figures 6A-6C, 6J-6L). Consistent with the in vitro results, the expressions of SNHG4 and RRM2 was found downregu-
A

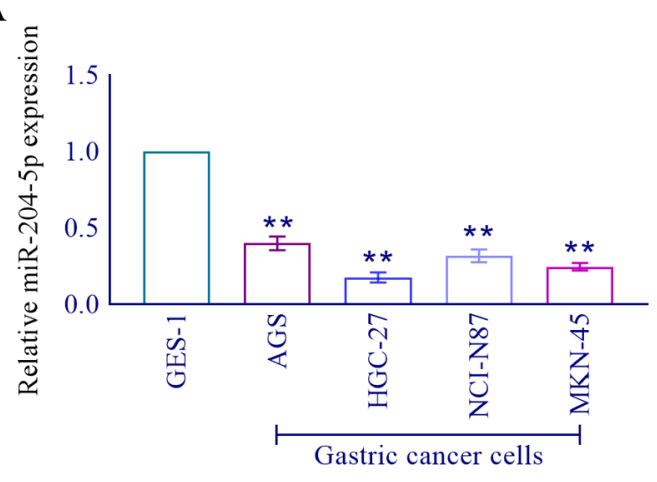

D

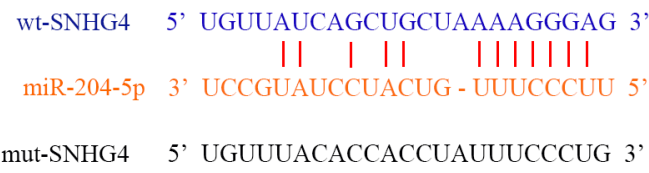

E

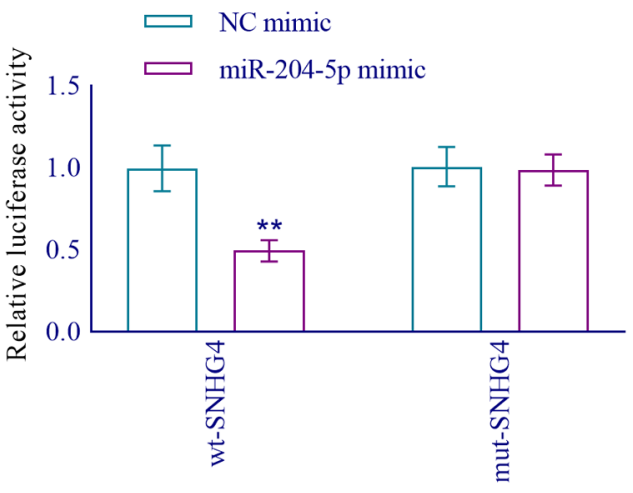

B

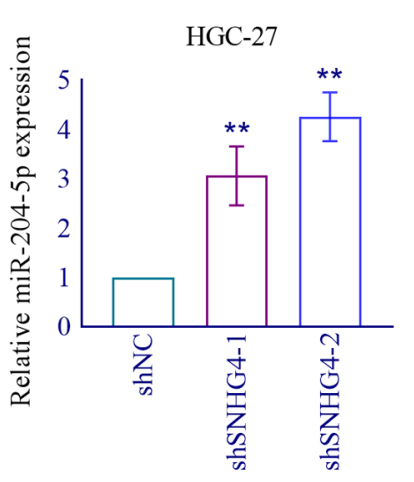

$\mathbf{F}$

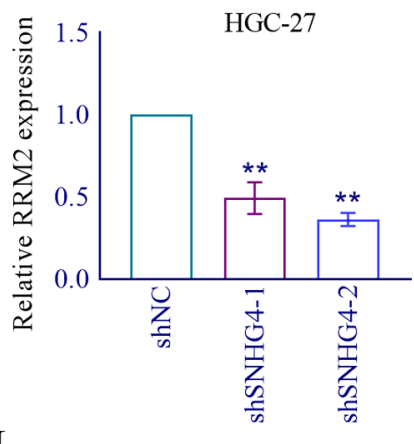

H

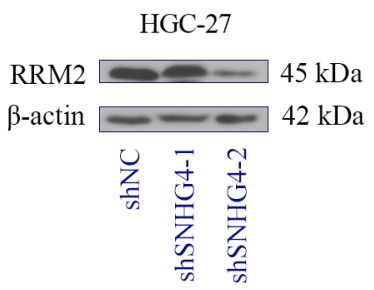

C

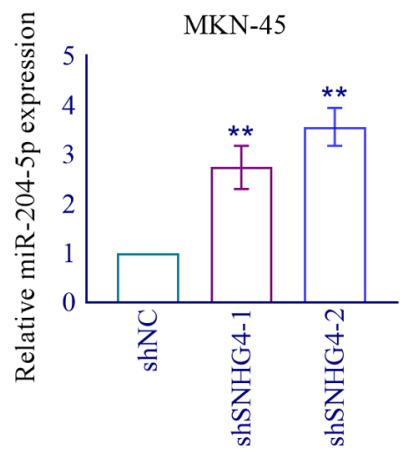

G

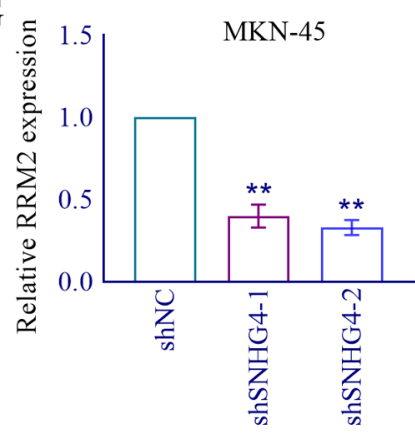

I

MKN-45

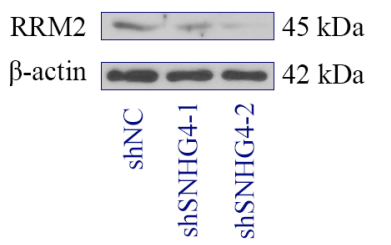

Figure 4. miR-204-5p is a target miRNA of SNHG4. A) The relative miR-204-5p expressions in human gastric mucosal epithelial cell line GES-1 and human GC cell lines AGS, HGC-27, NCI-N87, and MKN-45 were detected using qRT-PCT. B, C) Knockdown of SNHG4 increases miR-204-5p expressions in HGC-27 cells (B) and MKN-45 cells (C), as detected by qRT-PCR. D) Schematic illustration of the putative targeting site of miR-204-5p and SNHG4. E) The luciferase activities were detected by dual-luciferase reporter gene assay after co-transfection of wild-type (wt)-SNHG4 or mutant-type (mut)-SNHG4 and NC mimic or miR-204-5p mimic. F, G) Knockdown of SNHG4 decreases RRM2 expressions in HGC-27 cells (F) and MKN-45 cells (G), as detected by qRT-PCR. H, I) Knockdown of SNHG4 decreases RRM2 expressions in HGC-27 cells (H) and MKN-45 cells (I), as assessed by western blot. ${ }^{* *} \mathrm{p}<0.01$ 
A

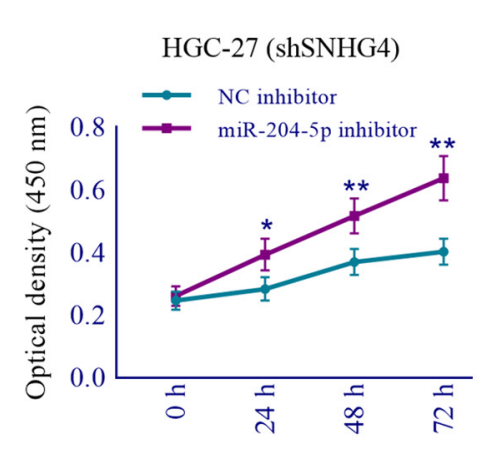

F

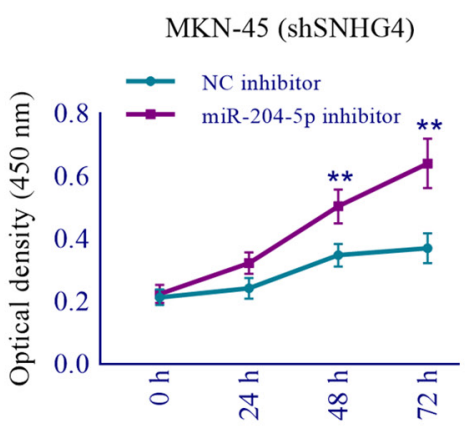

B

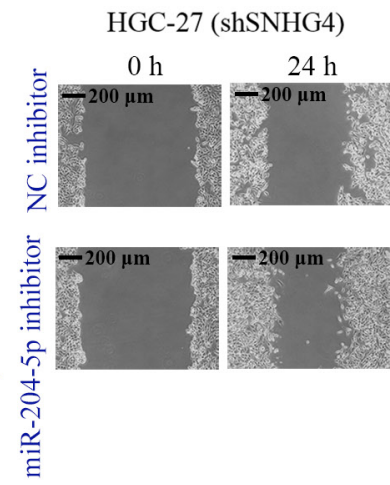

G

MKN-45 (shSNHG4)

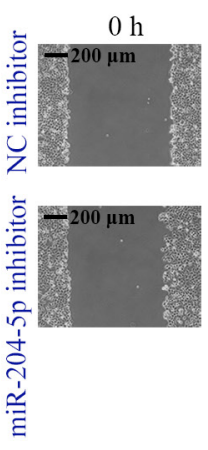

$24 \mathrm{~h}$

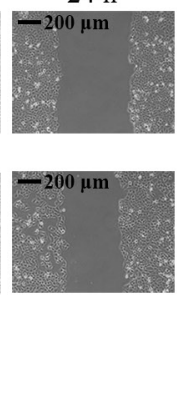

C

HGC-27 (shSNHG4)

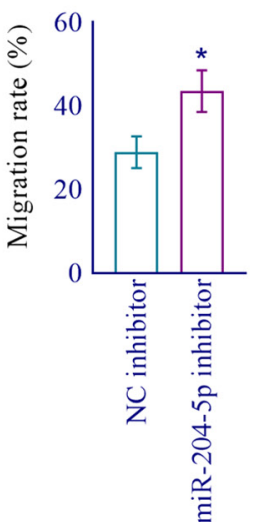

H
MKN-45 (shSNHG4) MKN-45 (shSNHG4)
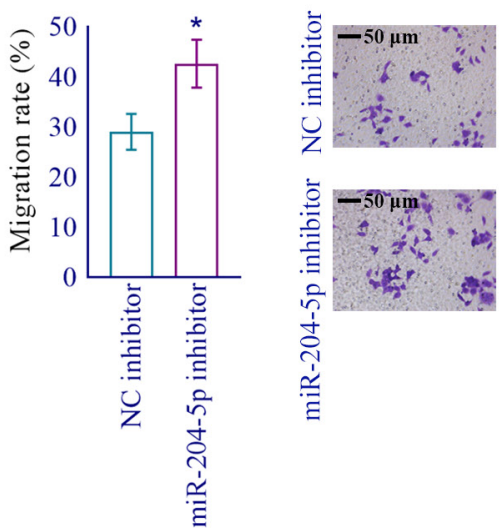

D

E
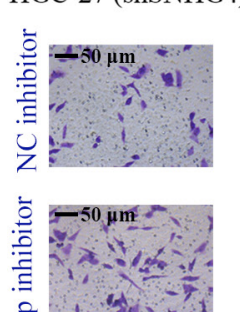

in

芒

J
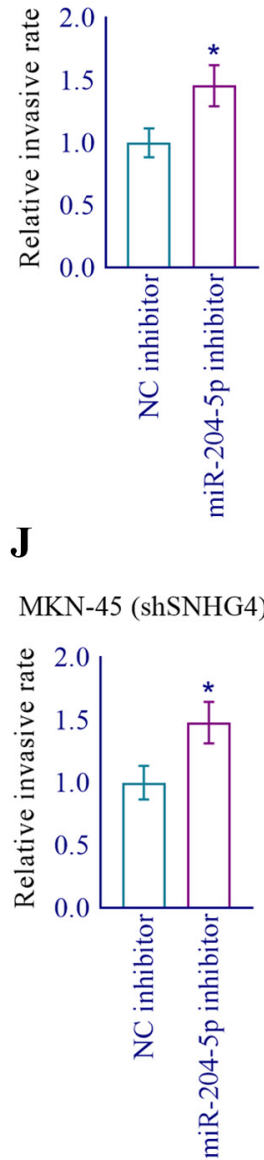

Figure 5. miR-204-5p inhibition promotes cell proliferation and metastasis in SNHG4-knockdown GC cells. A) miR-204-5p inhibition promotes cell proliferation in SNHG4-knockdown HGC-27 cells, as assessed by the CCK-8 assay. B, C) miR-204-5p inhibition promotes cell migration in SNHG4knockdown HGC-27 cells, as assessed by the wound-healing assay. D, E) miR-204-5p inhibition promotes cell invasion in SNHG4-knockdown HGC-27 cells, as assessed by the Transwell assay. F) miR-204-5p inhibition promotes cell proliferation in SNHG4-knockdown MKN-45 cells, as assessed by the CCK-8 assay. G, H) miR-204-5p inhibition promotes cell migration in SNHG4-knockdown MKN-45 cells, as assessed by the wound-healing assay. I, J) miR-204-5p inhibition promotes cell invasion in SNHG4-knockdown MKN-45 cells, as assessed by the Transwell assay. ${ }^{\star} \mathrm{p}<0.05$; ${ }^{\star *} \mathrm{p}<0.01$

lated, while the expression of miR-204-5p was found upregulated in the isolated tumor tissues (Figures 6D-6G, 6M-6P) of mice in the shSNHG4 groups. Similarly, the expressions of a proliferation marker Ki67, and two metastasis markers MMP2 and MMP9, were found downregulated in shSNHG4transfected GC cells (Figures 6H, 6J, 6Q, and 6R). These results showed that the knockdown of lncRNA SNHG4 successfully inhibited the GC cell growth in vivo.

\section{Discussion}

Until now, GC is still the leading cause of cancer-related death in both sexes worldwide, especially in Eastern Asia [18]. Despite some advances that have been made in GC treatment, including surgical resection and chemotherapy, the mortality of advanced GC patients still stays high due to the poor prognosis and recurrence. Notably, emerging evidence has revealed that lncRNAs, a pivotal group of ncRNAs, are intensely involved in the occurrence, metastasis, and recurrence of $\mathrm{GC}$, and might become compelling therapeutic targets for GC therapy. A novel lncRNA, SNHG4, has been documented as an important regulator in several types of tumor carcinogenesis and progression recently. However, the bio-functional role and underlying mechanism of SNHG4 in GC progression remain unclear.

In the present study, we found that SNHG4 was highly expressed in GC cell lines. To further investigate the role of SNHG4 in GC progression, we constructed two stable SNHG4-knockdown GC cell lines for the loss-of-function exploration. We found that the knockdown of SNHG4 
A

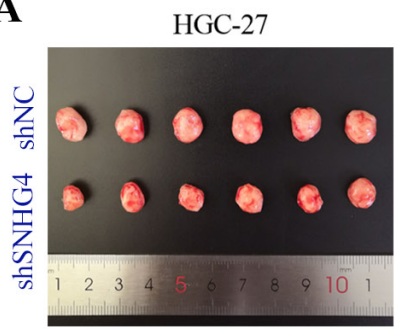

B

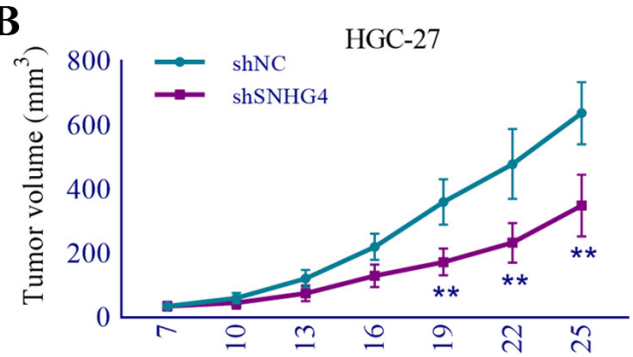

$\mathrm{C}$

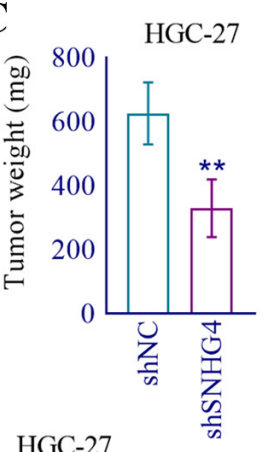

D

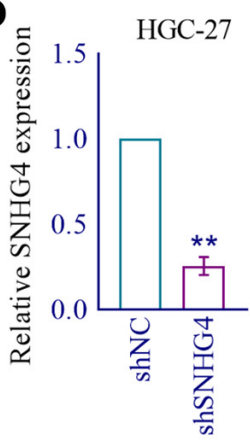

$\mathbf{E}$

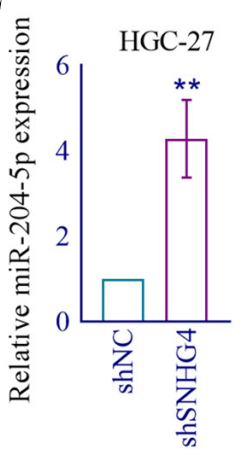

F

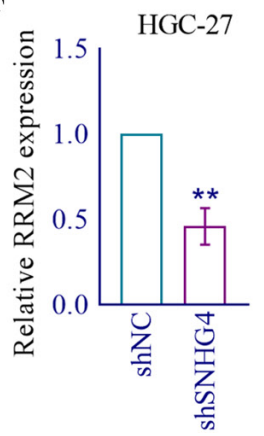

G

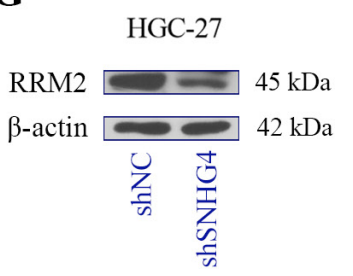

H

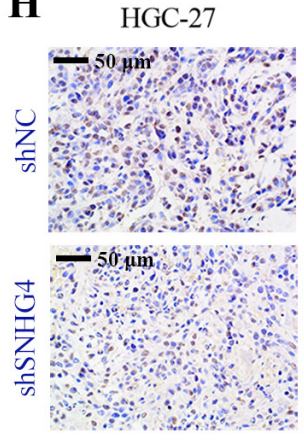

I

\section{HGC-27}

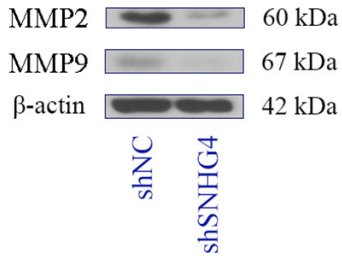

$\mathbf{J}$

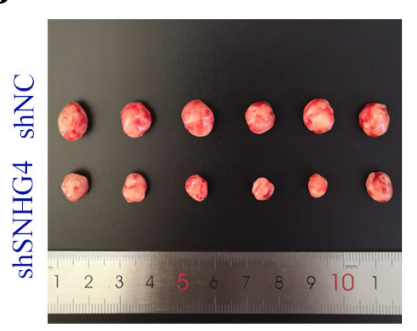

K

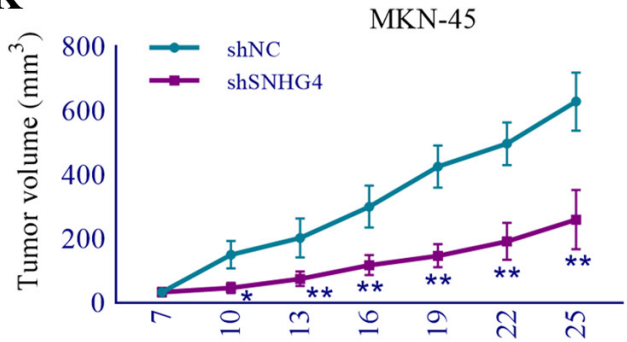

$\mathbf{L}$

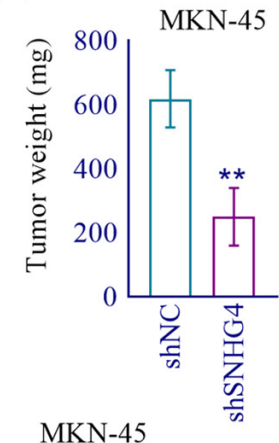

M

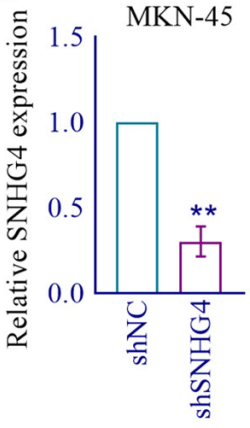

$\mathbf{N}$

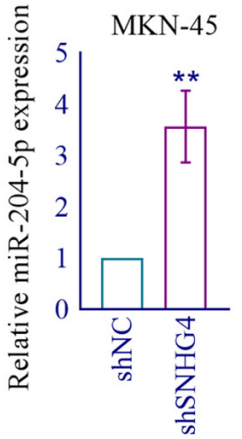

$\mathbf{O}$

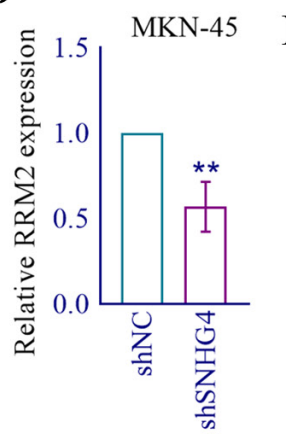

Q $\quad \mathrm{MKN}-45$

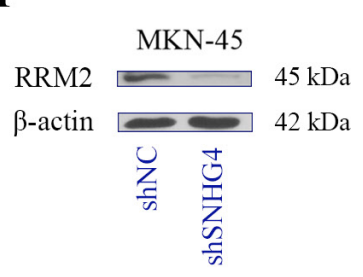

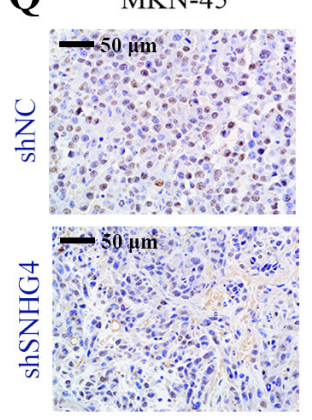

$\mathbf{R}$

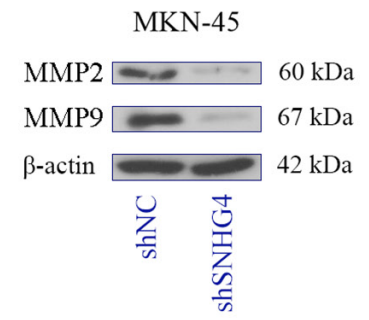

Figure 6. Knockdown of lncRNA SNHG4 inhibits GC cell growth in vivo. Mice were inoculated with stable shNC or shSNHG4 transfected HGC-27 or MKN-45 cells. A, J) Tumor images in mice inoculated with stable shNC or shSNHG4 transfected HGC-27 cells (A) and MKN-45 cells (J). B, K) Knockdown of SNHG4 decreases tumor volumes in a xenograft tumor model of HGC-27 cells (B) and MKN-45 cells (K). C, L) Knockdown of SNHG4 decreases tumor weights in a xenograft tumor model of HGC-27 cells (C) and MKN-45 cells (L). D, M) The relative SNHG4 expression in mice inoculated with stable shNC or shSNHG4 transfected HGC-27 cells (D) and MKN-45 cells (M), as assessed by qRT-PCR. E, N) Knockdown of SNHG4 increases miR-204-5p expression in a xenograft tumor model of HGC-27 cells (E) and MKN-45 cells (N), as assessed by qRT-PCR. F, O) Knockdown of SNHG4 decreases RRM2 expression in a xenograft tumor model of HGC-27 cells (F) and MKN-45 cells (O), as assessed by qRT-PCR. G, P) Knockdown of SNHG4 decreases RRM2 expression in a xenograft tumor model of HGC-27 cells (G) and MKN-45 cells (P), as assessed by western blot. H, Q) Knockdown of SNHG4 decreases Ki67 expression in a xenograft tumor model of HGC-27 cells (H) and MKN-45 cells (Q), as assessed by immunohistochemistry. I, R) Knockdown of SNHG4 decreases MMP2 and MMP9 expressions in a xenograft tumor model of HGC-27 cells (I) and MKN-45 cells (R), as assessed by western blot. ${ }^{*} \mathrm{p}<0.05 ;{ }^{* *} \mathrm{p}<0.01$ 
reduced cell viability and induced cell cycle arrest, accompanied by a decrease in the expressions of G1 checkpoint protein Cyclin D1, and its catalytic subunit CDK6 [19, 20]. Also, we found that SNHG4 knockdown reduced the expression of Ki67, a well-known proliferation marker that predicts cancer progression [21], indicating that SNHG4 knockdown successfully inhibited the proliferation capacity of GC cells.

Metastasis, a biological cascade that ultimately results in the widespread dissemination of cancer cells into multiple organs, is the main incentive of cancer mortality [22]. GC metastasis also has no exception [23]. It has been reported that the high level of SNHG4 was associated with lymph node metastasis in patients with prostate cancer [24], and also the distant metastasis in osteosarcoma patients [25]. Migration and invasion are two vital initial steps of cancer metastasis [26]. It has been illustrated that matrix metallopeptidases are important enzymes that function in degrading basement membrane type IV collagen [27], which is thought as the first step for cancer invasion and metastasis [28]. Consistent with these notions, our results showed that SNHG4 knockdown effectively suppressed GC cell migration and invasion abilities, accompanied by the decrease of two key matrix metallopeptidases MMP2 and MMP9, indicating that SNHG4 knockdown effectively inhibited the metastasis capacity of GC cells.

Moreover, recent evidence has revealed that lncRNAs regulate tumorigenesis by acting as ceRNAs to specific miRNAs. miRNAs are also a group of ncRNAs that play functional roles in regulating multiple biological processes through translational repression or mRNA degradation [29, 30]. By using online bioinformatics prediction software, we found that there is the binding potential between SNHG4 and miR-204-5p. miR-204-5p has been documented as a tumorsuppressive miRNA in GC via downregulating ubiquitinspecific peptidase 47 (USP47) and RAB22A [16]. Also, miR-204-5p was reported to inhibit GC cell proliferation and metastasis by targeting epidermal growth factor receptor (EGFR) [15]. A recent study reported that SNHG4 promotes tumorigenesis and invasion by sponging miR-204-5p in renal cell carcinoma [17]. Consistently, by performing rescue experiments and a dual-luciferase reporter gene assay, we demonstrated that SNHG4 affects GC proliferation and metastasis by regulating miR-204-5p in this work.

Notably, it has been documented that miR-204-5p affected the development of varied human tumors progression by regulating diverse genes such as runt-related transcription factor 2 (RUNX2) [17], human epidermal growth factor receptor 2 (HER-2) [31], high mobility group B1 (HMGB1) [32], and RRM2 [33]. Among them, RRM2 is a catalytic subunit of ribonucleotide reductase that is intensely involved in DNA synthesis [34]. Its oncogenic effects in GC progression have been identified as previously reported [35-37]. Importantly, research has indicated that inhibiting RRM2 may be a potential therapeutic strategy to overcome the resistance of chemotherapy [38]. Consistently in our work, we found that in SNHG4-knockdown GC cells, RRM2 presented a lower level. These results implied that SNHG4 may regulate GC progression by sponging miR-204-5p and un-regulating RRM2 expression.

Regrettably, in this work, there appear to be some deficiencies. This study lacks the support of the measurement of SNHG4 expression in clinical samples. Also, the regulatory correlation between SNHG4 and miR-204-5p needs to be further explored in GC. Despite it is hard for us to make better improvements in these aspects due to the existing constraints, the in-depth investigations are still worthy of our further research.

In the current study, we demonstrated that the knockdown of SNHG4 dramatically suppressed the proliferation and metastasis on GC cells via regulating miR-204-5p. Our findings first demonstrated that SNHG4 may serve as an oncogene in GC development and indicated that SNHG4 might become a potential therapeutic target in GC treatment.

\section{References}

[1] BRAY F, FERLAY J, SOERJOMATARAM I, SIEGEL RL, TORRE LA et al. Global cancer statistics 2018: GLOBOCAN estimates of incidence and mortality worldwide for 36 cancers in 185 countries. CA Cancer J Clin 2018; 68: 394-424. https://doi.org/10.3322/caac.21492

[2] FERRO A, PELETEIRO B, MALVEZZI M, BOSETTI C, BERTUCCIO $\mathrm{P}$ et al. Worldwide trends in gastric cancer mortality (1980-2011), with predictions to 2015, and incidence by subtype. Eur J Cancer 2014; 50: 1330-1344. https:// doi.org/10.1016/j.ejca.2014.01.029

[3] VAN CUTSEM E, SAGAERT X, TOPAL B, HAUSTERMANS K, PRENEN H. Gastric cancer. Lancet 2016; 388: 2654-2664. https://doi.org/10.1016/s0140-6736(16)30354-3

[4] PASECHNIKOV V, CHUKOV S, FEDOROV E, KIKUSTE I, LEJA M. Gastric cancer: prevention, screening and early diagnosis. World J Gastroenterol 2014; 20: 13842-13862. https://doi.org/10.3748/wjg.v20.i38.13842

[5] FIGUEIREDO C, CAMARGO MC, LEITE M, FUENTES-PANANá EM, RABKIN CS et al. Pathogenesis of Gastric Cancer: Genetics and Molecular Classification. Curr Top Microbiol Immunol 2017; 400: 277-304. https://doi. org/10.1007/978-3-319-50520-6_12

[6] ZHAO J, DU P, CUI P, QIN Y, HU C et al. LncRNA PVT1 promotes angiogenesis via activating the STAT3/VEGFA axis in gastric cancer. Oncogene 2018; 37: 4094-4109. https://doi. org/10.1038/s41388-018-0250-Z

[7] FENG Q, WU X, LI F, NING B, LU X et al. miR-27b inhibits gastric cancer metastasis by targeting NR2F2. Protein Cell 2017; 8: 114-122. https://doi.org/10.1007/s13238-0160340-Z

[8] ZHANG F, WANG XS, TANG B, LI PA, WEN Y et al. Long non-coding RNA FTX promotes gastric cancer progression by targeting miR-215. Eur Rev Med Pharmacol Sci 2020; 24: 3037-3048. https://doi.org/10.26355/eurrev_202003_20668 
[9] SHARMA D, LARRIERA AI, PALACIO-MANCHENO PE, GATTI V, FRITTON JC et al. The effects of estrogen deficiency on cortical bone microporosity and mineralization. Bone 2018; 110: 1-10. https://doi.org/10.1016/j.bone.2018.01.019

[10] XIONG X, FENG Y, LI L, YAO J, ZHOU M et al. Long non-coding RNA SNHG1 promotes breast cancer progression by regulation of LMO4. Oncol Rep 2020; 43: 1503-1515. https://doi.org/10.3892/or.2020.7530

[11] SHI J, DING W, LU H. Identification of Long Non-Coding RNA SNHG Family as Promising Prognostic Biomarkers in Acute Myeloid Leukemia. Onco Targets Ther 2020; 13: 84418450. https://doi.org/10.2147/ott.s265853

[12] XU R, FENG F, YU X, LIU Z, LAO L. LncRNA SNHG4 promotes tumour growth by sponging miR-224-3p and predicts poor survival and recurrence in human osteosarcoma. Cell Prolif 2018; 51: e12515. https://doi.org/10.1111/cpr.12515

[13] LI H, HONG J, WIJAYAKULATHILAKA W. Long noncoding RNA SNHG4 promotes cervical cancer progression through regulating c-Met via targeting miR-148a-3p. Cell Cycle 2019; 18: 3313-3324. https://doi.org/10.1080/1538410 1.2019 .1674071

[14] TAM C, WONG JH, TSUI SKW, ZUO T, CHAN TF et al. LncRNAs with miRNAs in regulation of gastric, liver, and colorectal cancers: updates in recent years. Appl Microbiol Biotechnol 2019; 103: 4649-4677. https://doi.org/10.1007/ s00253-019-09837-5

[15] WANG Y, ZHANG H, GE S, FAN Q, ZHOU L et al. Effects of miR-138-5p and miR-204-5p on the migration and proliferation of gastric cancer cells by targeting EGFR. Oncol Rep 2018; 39: 2624-2634. https://doi.org/10.3892/or.2018.6389

[16] ZHANG B, YIN Y, HU Y, ZHANG J, BIAN Z et al. MicroRNA-204-5p inhibits gastric cancer cell proliferation by downregulating USP47 and RAB22A. Med Oncol 2015; 32: 331. https://doi.org/10.1007/s12032-014-0331-y

[17] WU J, LIU T, SUN L, ZHANG S, DONG G. Long noncoding RNA SNHG4 promotes renal cell carcinoma tumorigenesis and invasion by acting as ceRNA to sponge miR-204-5p and upregulate RUNX2. Cancer Cell Int 2020; 20: 514. https:// doi.org/10.1186/s12935-020-01606-z

[18] RAHMAN R, ASOMBANG AW, IBDAH JA. Characteristics of gastric cancer in Asia. World J Gastroenterol 2014; 20: 4483-4490. https://doi.org/10.3748/wjg.v20.i16.4483

[19] QIE S, DIEHL JA. Cyclin D1, cancer progression, and opportunities in cancer treatment. J Mol Med (Berl) 2016; 94: 1313-1326. https://doi.org/10.1007/s00109-016-1475-3

[20] LANDIS MW, PAWLYK BS, LI T, SICINSKI P, HINDS PW. Cyclin D1-dependent kinase activity in murine development and mammary tumorigenesis. Cancer Cell 2006; 9: 13-22. https://doi.org/10.1016/j.ccr.2005.12.019

[21] YANG C, ZHANG J, DING M, XU K, LI L et al. Ki67 targeted strategies for cancer therapy. Clin Transl Oncol 2018; 20: 570-575. https://doi.org/10.1007/s12094-017-1774-3

[22] VALASTYAN S, WEINBERG RA. Tumor metastasis: molecular insights and evolving paradigms. Cell 2011; 147: 275-292. https://doi.org/10.1016/j.cell.2011.09.024
[23] TAN Z. Recent Advances in the Surgical Treatment of Advanced Gastric Cancer: A Review. Med Sci Monit 2019; 25: 3537-3541. https://doi.org/10.12659/msm.916475

[24] WANG ZY, DUAN Y, WANG P. SP1-mediated upregulation of lncRNA SNHG4 functions as a ceRNA for miR-377 to facilitate prostate cancer progression through regulation of ZIC5. J Cell Physiol 2020; 235: 3916-3927. https://doi. org/10.1002/jcp. 29285

[25] HUANG YF, LU L, SHEN HL, LU XX. LncRNA SNHG4 promotes osteosarcoma proliferation and migration by sponging miR-377-3p. Mol Genet Genomic Med 2020; 8: e1349. https://doi.org/10.1002/mgg3.1349

[26] TOCHHAWNG L, DENG S, PERVAIZ S, YAP CT. Redox regulation of cancer cell migration and invasion. Mitochondrion 2013; 13: 246-253. https://doi.org/10.1016/j. mito.2012.08.002

[27] WANG X, YANG B, SHE Y, YE Y. The lncRNA TP73-AS1 promotes ovarian cancer cell proliferation and metastasis via modulation of MMP2 and MMP9. J Cell Biochem 2018; 119: 7790-7799. https://doi.org/10.1002/jcb.27158

[28] NAKAJIMA M, WELCH DR, BELLONI PN, NICOLSON GL. Degradation of basement membrane type IV collagen and lung subendothelial matrix by rat mammary adenocarcinoma cell clones of differing metastatic potentials. Cancer Res 1987; 47: 4869-4876.

[29] CHAN JJ, TAY Y. Noncoding RNA: RNA Regulatory Networks in Cancer. Int J Mol Sci 2018; 19. https://doi. org/10.3390/ijms19051310

[30] HUNTZINGER E, IZAURRALDE E. Gene silencing by microRNAs: contributions of translational repression and mRNA decay. Nat Rev Genet 2011; 12: 99-110. https://doi. org/10.1038/nrg2936

[31] YANG S, CHEN B, ZHANG B, LI C, QIU Y et al. miR-204-5p promotes apoptosis and inhibits migration of gastric cancer cells by targeting HER-2. Mol Med Rep 2020; 22: 2645-2654. https://doi.org/10.3892/mmr.2020.11367

[32] LU M, QIN X, ZHOU Y, LI G, LIU Z et al. LncRNA HOTAIR suppresses cell apoptosis, autophagy and induces cell proliferation in cholangiocarcinoma by modulating the miR-2045p/HMGB1 axis. Biomed Pharmacother 2020; 130: 110566. https://doi.org/10.1016/j.biopha.2020.110566

[33] LIANG WH, LI N, YUAN ZQ, QIAN XL, WANG ZH. DSCAM-AS1 promotes tumor growth of breast cancer by reducing miR-204-5p and up-regulating RRM2. Mol Carcinog 2019; 58: 461-473. https://doi.org/10.1002/mc.22941

[34] MAZZU YZ, ARMENIA J, CHAKRABORTY G, YOSHIKAWA Y, COGGINS SA et al. A Novel Mechanism Driving Poor-Prognosis Prostate Cancer: Overexpression of the DNA Repair Gene, Ribonucleotide Reductase Small Subunit M2 (RRM2). Clin Cancer Res 2019; 25: 4480-4492. https:// doi.org/10.1158/1078-0432.ccr-18-4046

[35] KANG W, TONG JH, CHAN AW, ZHAO J, WANG S et al. Targeting ribonucleotide reductase M2 subunit by small interfering RNA exerts anti-oncogenic effects in gastric adenocarcinoma. Oncol Rep 2014; 31: 2579-2586. https://doi. org/10.3892/or.2014.3148 
[36] MORIKAWA T, HINO R, UOZAKI H, MAEDA D, USHIKU T et al. Expression of ribonucleotide reductase M2 subunit in gastric cancer and effects of RRM2 inhibition in vitro. Hum Pathol 2010; 41: 1742-1748. https://doi.org/10.1016/j. humpath.2010.06.001
[37] ZHONG Z, CAO Y, YANG S, ZHANG S. Overexpression of RRM2 in gastric cancer cell promotes their invasiveness via AKT/NF- $\kappa B$ signaling pathway. Pharmazie 2016; 71:280-284.

[38] ZHAN Y, JIANG L, JIN X, YING S, WU Z et al. Inhibiting RRM2 to enhance the anticancer activity of chemotherapy. Biomed Pharmacother 2021; 133: 110996. https://doi. org/10.1016/j.biopha.2020.110996 